\title{
Impact of cancer on use of glucose-lowering drug treatment in individuals with diabetes: potential mechanisms. Reply to Pouwer F [letter]
}

\author{
Marjolein M. J. Zanders ${ }^{1}$ - Harm R. Haak ${ }^{2,3,4}$ - Myrthe P. P. van Herk-Sukel ${ }^{5}$ • \\ Lonneke V. van de Poll-Franse ${ }^{1,6}$ • Jeffrey A. Johnson ${ }^{7}$
}

Received: 6 March 2015 / Accepted: 17 March 2015 /Published online: 3 April 2015

(C) Springer-Verlag Berlin Heidelberg 2015

Keywords Adherence $\cdot$ Cancer $\cdot$ Glucose-lowering drugs

To the Editor: We thank Frans Pouwer for his comments on our study [1] exploring adherence to glucose-lowering drugs following a cancer diagnosis among individuals with diabetes [2]. Our findings revealed that there was a significant drop in the medication possession ratio at the time of cancer diagnosis, with the largest drops among patients with stage IV disease and gastrointestinal or pulmonary cancers. In his letter, Pouwer describes two potential mechanisms that could explain these results [1].

As Pouwer indicates, weight loss has been associated with improvements in beta cell function and insulin sensitivity

Marjolein M. J. Zanders

m.zanders@iknl.nl

1 Netherlands Comprehensive Cancer Organisation, P.O. Box 231, 5600 AE Eindhoven, the Netherlands

2 Department of Internal Medicine, Máxima Medical Centre, Eindhoven/Veldhoven, the Netherlands

3 Department of Internal Medicine, Division of General Internal Medicine, Maastricht University Medical Centre+, Maastricht, the Netherlands

4 Department of Health Services Research, CAPHRI School for Public Health and Primary Care, Maastricht University, Maastricht, the Netherlands

5 PHARMO Institute for Drug Outcomes Research, Utrecht, the Netherlands

6 CoRPS-Center of Research on Psychology in Somatic Diseases, Department of Medical and Clinical Psychology, Tilburg University, Tilburg, the Netherlands

7 School of Public Health, University of Alberta, Edmonton, AB, Canada among individuals with diabetes. As a result, glucose levels decline, $\mathrm{HbA}_{1 \mathrm{c}}$ values improve and less glucose-lowering drug treatment may be required to obtain optimal metabolic control. While weight loss is common among cancer patients, especially among those with gastrointestinal, lung or advanced cancer, as described by Pouwer, the impact of weight loss on metabolic control and thus on diabetes treatment among these cancer patients is, to our knowledge, unknown. However, according to our research, $\mathrm{HbA}_{1 \mathrm{c}}$ values improve before colorectal cancer diagnosis (M. M. J. Zanders, M. P. P. van Herk-Sukel, R. M. C. Herings, L. V. van de Poll-Franse, H. R. Haak, unpublished data), and it is possible that this might be the result of weight loss. Although we might expect that the decisions made regarding diabetes treatment are similar for cancer patients and those without cancer (i.e. improvement in $\mathrm{HbA}_{1 \mathrm{c}}$ leads to reductions in glucose-lowering drug dose), we assume then that the attention of patient and physician is also on metabolic control and diabetes treatment around cancer diagnosis. In addition, in practice, clinical decision-making regarding metabolic control is based on anticipating an interplay of factors besides weight loss, such as food intake, glucose values, $\mathrm{HbA}_{1 \mathrm{c}}$, risk of hypoglycaemia, diarrhoea, and presence of diabetes-related complications, such as kidney dysfunction and foot lesions [3]. Finally, physicians might decide to not change diabetes treatment among cancer patients if they find that $\mathrm{HbA}_{1 \mathrm{c}}$ values increase (i.e. worsen) back to baseline after cancer diagnosis, as our research suggests (M. M. J. Zanders, M. P. P. van Herk-Sukel, R. M. C. Herings, L. V. van de Poll-Franse, H. R. Haak, unpublished data). Nevertheless, weight loss might be one of the multiple factors that make physicians decide to lower the dose or even discontinue diabetes treatment. When weight loss causes physicians to verbally inform patients to change their daily regimen, the same amount of treatment (already in the patient's possession) might be distributed over a greater 
number of days, giving the impression that adherence has declined when in fact it hasn't. If this was the most plausible and only explanation for the study findings, then, as suggested by Pouwer, we should have used the words 'medication use' instead of 'medication adherence'.

In our manuscript we briefly described that stress following a major event could have contributed to the observed decline in adherence. Depressive symptoms might have a role in this, since depression is related to stress and seems also to be associated with lower adherence rates, as discussed in the comment of Pouwer [1]. Consequently, it would be very relevant and interesting to investigate in a future study whether the decline in adherence was modified by the presence of depression or depressive symptoms among patients with cancer and diabetes.

As elaborated in our study [2] and in the contribution by Pouwer [1], we can conclude that the association between adherence to glucose-lowering treatment and cancer is probably explained by an interplay of factors. We thank Pouwer for his valuable additions regarding potential mechanisms to explain the complex association. The explanation for the decline in adherence should be studied in more detail, taking into account the discussed mechanisms and the type of cancer.

Funding The present research was supported by a grant from the European Foundation for the Study of Diabetes (EFSD).

Duality of interest The authors declare that there is no duality of interest associated with this manuscript.

Contribution statement All authors were responsible for the conception and design of the manuscript, drafting the response letter and revising it critically for important intellectual content. All authors approved the version to be published.

\section{References}

1. Pouwer F (2015) Impact of cancer on use of glucose-lowering drug treatment in individuals with diabetes: potential mechanisms. Diabetologia. doi:10.1007/s00125-015-3560-5

2. Zanders MM, Haak HR, van Herk-Sukel MP, van de Poll-Franse LV, Johnson JA (2015) Impact of cancer on adherence to glucose-lowering drug treatment in individuals with diabetes. Diabetologia 58:951-960

3. American Diabetes Association (2014) Standards of medical care in diabetes-2014. Diabetes Care 37(Suppl 1):S14-S80 